\title{
EDITORIAL
}

\section{Endoscopic ultrasound-guided biopsy in the chest: little to lose, much to gain}

\author{
P. Vilmann and S.S. Larsen
}

$\mathbf{T}$ issue diagnosis of pathological lesions located in the mediastinum has been difficult to obtain by nonsurgical methods, well protected as it is by the bony covering of the thorax and the surrounding lungs. A variety of lesions involve the mediastinum either as primary tumours or as secondary deposits from both benign and malignant diseases. It is therefore of paramount clinical importance to develop safe and accurate minimally invasive methods that are able to tissue diagnose such lesions in order to plan specific treatments.

The most frequent cancer involving the mediastinum is nonsmall cell lung cancer (NSCLC) accounting for $\sim 80 \%$ of lung cancers. The prognosis of NSCLC is poor and closely related to the stage of disease. NSCLC usually metastasises first to hilar and mediastinal lymph nodes. Subsequently, haematogenous metastasis to distant sites may occur. The treatment of NSCLC is stage dependent, and is in general by either surgical resection, provided no spread outside the lung is found (stage I and II), or induction chemotherapy followed by resection, in patients with ipsilateral mediastinal lymph node metastases (stage IIIA), or chemo-/radiotherapy alone, provided contralateral mediastinal- or distant metastases are present (stage IIIB and IV).

When traditional pre-operative staging methods are used, $\sim 10 \%$ of operations for NSCLC result in explorative thoracotomy without tumour resection, due to advanced mediastinal disease not detected pre-operatively. In addition, 25-35\% of the apparently curative resections are unsuccessful because of early post-operative recurrent disease [1]. Therefore, surgery may be considered futile or unnecessary in up to $45 \%$ of patients operated on, apparently because the stage of the disease is more advanced than expected pre-operatively. It is therefore important to develop methods that more accurately stage this disease.

A growing number of publications have firmly established endoscopic ultrasound-guided biopsy as perhaps the most sensitive method in the diagnosis of posterior mediastinal lymph nodes and suspicious lesions. In expert hands the sensitivity of endoscopic ultrasound-guided fine-needle aspiration biopsy (EUS-FNA) for these lesions reaches 90$98 \%$. The number of patients included in the publications is currently $>1,500$ patients [2]. In this issue of European Respiratory Journal, CADDY et al. [3] further underline the benefit of EUS-FNA in mediastinal staging of selected patients with NSCLC and for diagnosis of unexplained mediastinal

CORRESPONDENCE: P. Vilmann, Gentofte University Hospital, 2900 Hellerup, Denmark. Fax: 45 39777629. E-mail: pevi@gentoftehosp.kbhamt.dk lymph nodes. The accuracy for mediastinal malignancy in this study with 52 consecutive patients was $95 \%$, comparable to that of other similar studies.

The reported complication rate of EUS-FNA is $<0.5 \%$, most of these were minor [2]. This is in contrast with the more invasive method of mediastinoscopy, with a reported complication rate of $2-5 \%$ and with the potential of fairly serious complications. In contrast to mediastinoscopy, the EUS-FNA procedure is performed with the patient in conscious sedation, in an outpatient setting.

One limitation of EUS-FNA in the chest is the ability to reach the region anterior to the trachea and main bronchi. Therefore, mediastinoscopy and EUS-FNA are at present considered as complementary methods, even though no studies have actually compared the two methods in a controlled and blinded design; whether one of the two methods may obviate or reduce the need for the other is unknown. SERNA et al. [4] compared mediastinoscopy with EUS-FNA in a retrospective study using different groups of patients and reported a sensitivity of EUS-FNA and mediastinoscopy of 86 and $100 \%$, respectively. This is in contradiction to our own experience [5] where mediastinoscopy was prospectively compared with EUS-FNA in an unselected cohort of 60 patients with NSCLC considered for surgery. Mediastinoscopy and EUS-FNA were conclusive for paratracheal or subcarinal mediastinal disease in six and 24 patients, respectively (sensitivity $24 / 96 \%$ ). These results suggest that mediastinoscopy is significantly inferior to EUS-FNA for staging of NSCLC patients, but final conclusions have to await controlled and blinded studies.

When looking critically at EUS-FNA results, most of the publications on EUS-FNA in lung cancer patients are retrospective studies with patients selected by computed tomography (CT), i.e. most patients had either lymph nodes $>1 \mathrm{~cm}$ or large solid lesions of unknown origin, most of these being lung cancers located adjacent to the oesophagus. Therefore, it seems that a selection bias may be present in many of these studies. If all NSCLC patients considered for surgery undergo EUS-FNA irrespective of $\mathrm{CT}$, how will this affect the staging accuracy? In addition to this, most of the published results are from expert centres. If EUS-FNA is taken up by all groups involved in lung cancer staging, how would this affect the diagnostic yield? We are not able to make firm conclusions at present. Recent studies have shown that CT-negative patients, i.e. patients without enlarged lymph nodes on CT, have lymph node metastases detected by EUS-FNA in 25\% of cases [6]. In other words, one of four lung cancer patients without enlarged lymph nodes in the 
mediastinum will have their management plan changed if EUS is performed routinely as the initial invasive staging modality.

There is also growing evidence that position emission tomography (PET)-positive patients should undergo EUS-FNA due to a false-positive rate of PET ranging 9-39\% [7]. A recent retrospective study in 104 patients with NSCLC found EUS-FNA to be significantly more accurate $(97 \%)$ than PET and CT for posterior mediastinal lymph nodes staging [7]. In another blinded comparative study in 79 patients with NSCLC between EUS-FNA, PET and CT, EUS-FNA and PET had a comparable sensitivity, but EUS-FNA had a superior specificity. Both methods had a sensitivity superior to that of CT [8, 9]. Falsepositive diagnoses were found by PET, CT and EUS-FNA in nine of 36, three of 20 and zero of 25 patients, respectively. Therefore, EUS-FNA was significantly the most reliable method to conclude inoperability.

Concerning mediastinal restaging after induction chemotherapy for NSCLC patients with verified N-2 disease, a single study has clearly demonstrated the benefit of EUS-FNA [10]. It was concluded that EUS-FNA seems able to identify the subgroup of down staged patients, who may benefit most from further surgical treatment. From the latter group, this issue of European Respiratory Journal provides us with a study of EUSFNA for the diagnosis of sarcoidosis after a nondiagnostic bronchoscopy [11]. Their results confirm the results of another large study of 124 patients with suspected sarcoidosis [12].

When all cons and pros concerning EUS-FNA in the chest are considered, it is difficult to maintain scepticism. More studies to see to what extend the less invasive method of EUS-FNA may replace mediastinoscopy are needed. Or are they? The same method as used via the oesophagus is at present tested with new instruments of reduced size via the trachea and main bronchi (endobronchial ultrasound-guided transbronchial needle aspiration biopsy (EBUS-TBNA)) [13]. Although the experience with EBUS-TBNA is limited at present, the results seem promising. Our own preliminary experience with a prototype EBUS-TBNA bronchoscope (Olympus, XBF-UC40P, Tokyo, Japan) in 285 patients has revealed a sensitivity of $\sim 85 \%$ for diagnosis of mediastinal lymphadenophathy suspected of malignancy (Unpublished data). RiNTOUL et al. [14] have also, in this issue of European Respiratory Journal, added important knowledge regarding the combination of EUS-FNA and EBUS-TBNA. Their results support that an improved staging of the mediastinum and hilar regions can be obtained when EUS-FNA and EBUS-TBNA are used in combination. More studies are needed before final conclusions on the combined approach can be made.

But how can we proceed to implement EUS-FNA as a routine procedure in respiratory medicine? At present most groups performing EUS-FNA in the chest are still gastroenterologists, since the method was originally developed in this specialty. This fact may also explain why EUS-FNA has not been more broadly taken up by the clinicians. However, it seems logical that the procedure will be taken over by chest physicians after the initial phase has passed. Until this time, collaboration between gastroenterologists and pulmonologists will continue, but the time is up for the national and international societies to wake-up and take over the responsibility for the implementation of EUS-FNA and possibly endobronchial ultrasound-guided transbronchial needle aspiration biopsy in respiratory and/or thoracic surgery. We really do not need additional proof before EUS-FNA is considered the gold standard for invasive staging of nonsmall cell lung cancer and for diagnosis of posterior mediastinal lesions; there is little to lose and much to gain.

\section{REFERENCES}

1 Shields TW. Surgical treatment of non-small cell bronchial carcinoma. In: Shields TW, ed. General Thoracic Surgery, 4th Edn. USA, Williams and Wilkins, 1994; 2, pp. 1159-1169.

2 Vilmann P, Larsen SS, Krasnik M. EUS-guided FNA for mediastinal tumors (lung cancer and lymph nodes). Digestive Endosc 2004; 16: 185-192.

3 Caddy G, Conron M, Wright G, Desmond P, Hart D, Chen RY. The accuracy of EUS-FNA in assessing mediastinal lymphadenopathy and staging patients with NSCLC. Eur Respir J 2005; 25: 410-415.

4 Serna DL, Aryan HE, Chang KJ, et al. An early comparison between endoscopic ultrasound-guided fine-needle aspiration and mediastinoscopy for diagnosis of mediastinal malignancy. Am Surg 1998; 64: 1014-1018.

5 Larsen SS, Viemann P, Dirksen A, Clementsen P, Jacobsen GK. Endoscopic ultrasound guided biopsy versus mediastinoscopy for analysis of paratracheal and subcarinal lymph nodes in lung cancer staging. Lung Cancer 2005; (In Press).

6 Wallace MB, Ravenel J, Block MI, et al. Endoscopic ultrasound in lung cancer patients with a normal mediastinum on computed tomography. Ann Thorac Surg 2004; 77: 1763-1768.

7 Eloubeidi MA, Cerfolio RJ, Chen VK, Desmond R, Syed S, Buddhiwardhan O. Endoscopic ultrasound-guided fine needle aspiration of mediastinal lymph node in patients with suspected lung cancer after positron emission tomography and computed tomography scans. Ann Thorac Surg 2005; 79: 263-268.

8 Fritscher-Ravens A, Davidson BL, Hauber HP, et al. Endoscopic ultrasound, positron emission tomography, and computerized tomography for lung cancer. Am J Respir Crit Care Med 2003; 168: 1293-1297.

9 Fritscher-Ravens A, Bohuslavizki $\mathrm{KH}$, Brandt L, et al. Mediastinal lymph node involvement in potentially resectable lung cancer: comparison of $\mathrm{CT}$, positron emission tomography, and endoscopic ultrasonography with and without fine-needle aspiration. Chest 2003; 123: 442-451.

10 Annema JT, Veselic M, Versteegh MI, Willems LN, Rabe KF. Mediastinal restaging: EUS-FNA offers a new perspective. Lung Cancer 2003; 42: 311-318.

11 Annema JT, Veselic M, Rabe KF. Endoscopic ultrasoundguided fine-needle aspiration for the diagnosis of sarcoidosis. Eur Respir J 2005; 25: 405-409.

12 Wildi SH, Judson MA, Fraig M. Is endosonography guided fine needle aspiration for sarcoidosis as good as we think? Thorax 2004; 59: 794-799.

13 Krasnik M, Vilmann P, Larsen SS, Jacobsen GK. Preliminary experience with a new method of endoscopic transbronchial real-time ultrasound guided biopsy for diagnosis of mediastinal and hilar lesions. Thorax 2003; 58: 1083-1086.

14 Rintoul RC, Skwarski KM, Murchison JT, Wallace WA, Walker WS, Penman ID. Endobronchial and endoscopic ultrasound-guided real-time fine-needle aspiration for mediastinal staging. Eur Respir J 2005; 25: 416-421. 